\title{
SKINmages
}

\section{An Uncommon Presentation Of Erosive Pustular Dermatosis: A Large, Crusted Plaque On The Scalp}

\author{
James MacKenzie, MS, ${ }^{1}$ Miriam Sagher, FNP-BC, ${ }^{2}$ Ethan Sagher, MD, ${ }^{2}$ Ben J. Friedman, MD, ${ }^{2}$ \\ Jesse Veenstra, MD, $\mathrm{PhD}^{2}$ \\ ${ }^{1}$ Wayne State University School of Medicine, Detroit, MI \\ ${ }^{2}$ Department of Dermatology, Henry Ford Health System, Detroit, MI
}
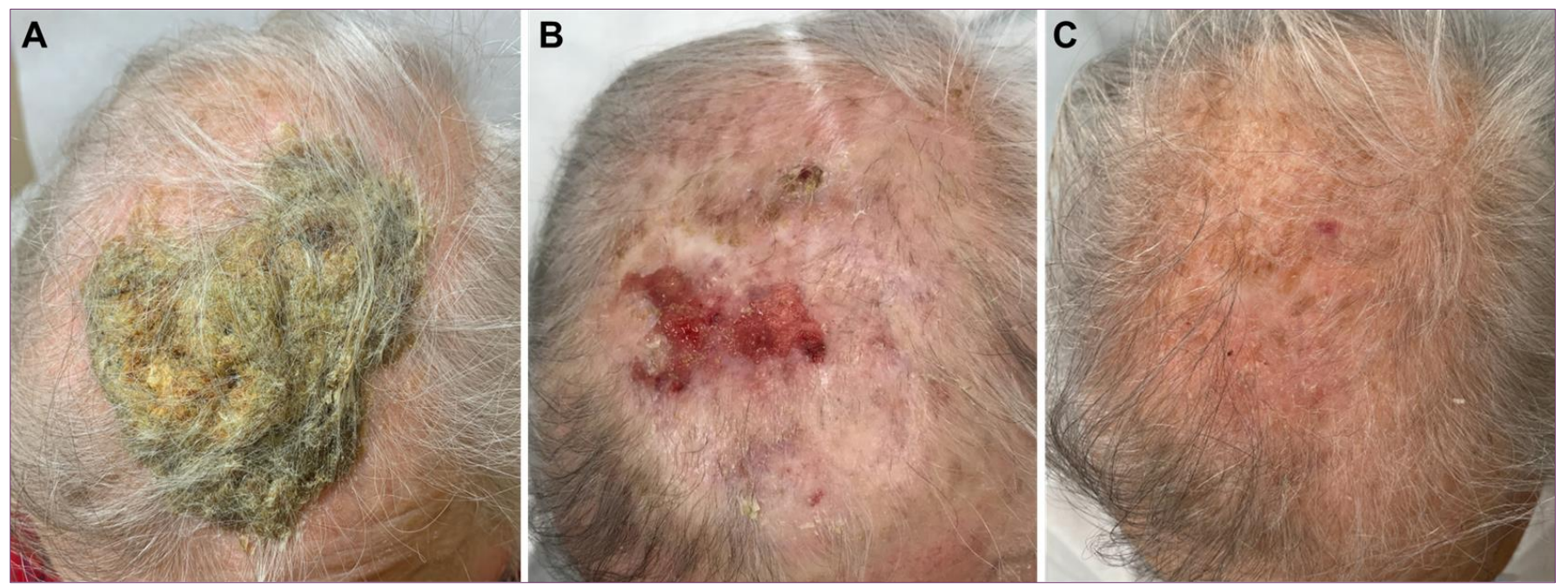

Figure 1. A) Large crusted plaque on the frontal scalp. B) Immediately after complete debridement. C) Complete resolution of affected area 6 weeks after debridement and clobetasol $0.05 \%$ ointment twice daily for 2 weeks.

Erosive pustular dermatosis (EPD) is an often underrecognized inflammatory condition that typically manifests with small scattered crusted erosions and pustules on the scalp of elderly patients. Many patients with EPD have concurrent actinic damage and a history of keratinocytic carcinomas, which may confound the diagnosis of EPD. Recognition of possible manifestations of EPD is imperative to ensure appropriate diagnostic workup and subsequent management to avoid non-productive therapy. We present an uncommon, but important to recognize, manifestation of
EPD as a large solitary crusted plaque on the scalp of an elderly patient, which has been seldom reported.

An 83-year-old Caucasian male presented with an asymptomatic crusted plaque on the scalp that had been growing over the past 5 years. On physical exam, there was a nontender $8 \times 8 \mathrm{~cm}$ thick crusted plaque overlying the frontal scalp (Fig 1A) without adjacent lesions. There was no palpable auricular or cervical lymphadenopathy present. The posterior section of the crust was removed with a scalpel to uncover an 
area of underlying friable, ulcerated tissue and purulent exudate where a wound culture and tangential shave biopsy were performed. Histopathology revealed a dense dermal infiltrate composed of lymphocytes, plasma cells, and neutrophils (Fig 2). Bacterial culture grew pan-sensitive

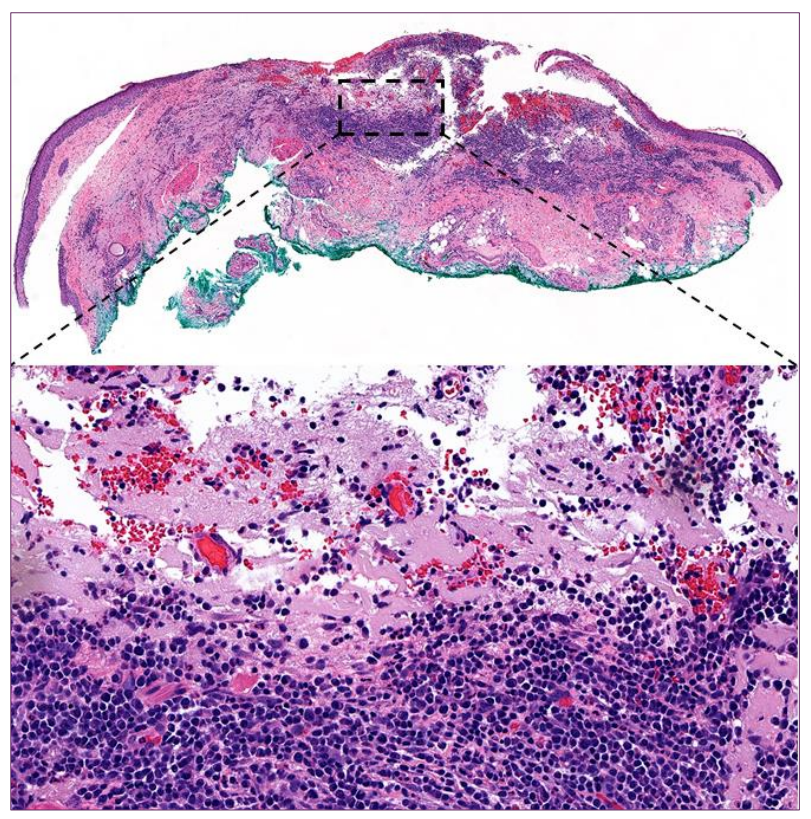

Figure 2. Low- and high-power magnification of shave biopsy showing ulceration with a dense dermal infiltrate composed of lymphocytes, plasma cells, and neutrophils (hematoxylin \& eosin)

staphylococcus aureus, which was treated with doxycycline $100 \mathrm{mg}$ twice daily for 14 days. Clinicopathological correlation led to a diagnosis of EPD (plaque type), and the patient underwent complete debridement of the crusted plaque revealing ulceration (Fig 1B). The patient was started on clobetasol $0.05 \%$ ointment twice daily to the ulcerated portion of the lesion with complete resolution after several weeks of use, as reepithelization was noted when he returned to the clinic 6 weeks after debridement (Fig $1 \mathrm{C})$. No recurrence was observed after 6 months.

Rarely, large solitary crusted plaques may be the sole manifestation of EPD, but have been seldom described in the current literature. ${ }^{1}$ The true prevalence and incidence of EPD are not well characterized but are likely underreported due to similar presentation to other diseases and subsequent misdiagnosis. The misdiagnosis or delayed diagnosis of patients with EPD is often fueled by a history of or coexistence of actinic keratoses and other keratinocytic carcinomas. $^{2}$ These diagnoses have led to months of non-productive treatment consequently prescribed to the patients, such as imiquimod and 5-fluorouracil, which may further contribute to the progression of EPD without effectively treating the underlying condition. ${ }^{3,4}$

The pathology of EPD occurs below the crust, which is why our patient returned to the office for complete debridement of the crusted plaque (Figure 1B) after a diagnosis of EPD was made. The histopathology of EPD is often variable and non-specific, highlighting the importance of clinicopathological correlation. ${ }^{5}$ Despite the lack of pathognomonic pathology, a biopsy remains essential to help rule out other differential entities, including malignant processes, infective, inflammatory (i.e., folliculitis decalvans), autoimmune (i.e., pemphigus, cicatricial pemphigoid, discoid lupus erythematosus), and psychosomatic. ${ }^{1}$ Additionally, a wound culture is also recommended as concurrent bacterial infection is common with EPD and should be treated, as in this case. ${ }^{1,6}$ Superpotent topical corticosteroids have historically been first-line treatment for EPD but are often used with variable success as refractory disease is not uncommon. ${ }^{1,5}$

The presentation of EPD in this case illustrates the requirement for thoughtful workup and clinicopathological correlation given the significant overlap that occurs in patient demographics, risk factors, and 
lesion morphology with keratinocytic carcinomas and EPD. Therefore, EPD must remain an important diagnostic consideration in elderly patients with lesions suspicious of cutaneous malignancies, as proper workup can potentially spare the patient weeks of non-productive therapy and optimize recovery.

Conflict of Interest Disclosures: None

Funding: None

Corresponding Author: Jesse Veenstra, MD, PhD Department of Dermatology Henry Ford Health System 3031 W. Grand Blvd, Ste 800 Detroit, MI 48202 jjveenst1@gmail.com

References:

1. Yeh R, Polcz M, Wong D. Erosive pustular dermatosis of the scalp - an Australian perspective: Insights to aid clinical practice. Australas J Dermatol 2019; 60: e272-e8.

2. Piccolo V, Russo T, Bianco $S$ et al. Erosive Pustular Dermatosis of the Scalp: Why Do We Miss It? Dermatology (Basel, Switzerland) 2019; 235: 390-5.

3. Corradin MT, Forcione M, Giulioni E et al. Erosive pustular dermatosis of the scalp induced by imiquimod. Case Rep Dermatol Med 2012; 2012: 828749.

4. Rongioletti F, Delmonte S, Rossi ME et al. Erosive pustular dermatosis of the scalp following cryotherapy and topical tretinoin for actinic keratoses. Clin Exp Dermatol 1999; 24: 499-500.

5. Starace $M$, Alessandrini $A$, Baraldi $C$ et al. Erosive pustular dermatosis of the scalp: challenges and solutions. Clin Cosmet Investig Dermatol 2019; 12: 691-8.

6. Thuraisingam T, Mirmirani P. Erosive Pustular Dermatosis: A Manifestation of Immunosenescence A Report of 8 Cases. Skin Appendage Disord 2018; 4: 180-6. 